Verena REMBOWSKI, Saarbrücken

\title{
Begriffsbilder und -konventionen in Begriffsfeldern: Was ist ein Würfel?
}

\section{Begriffsklärung}

Ein Beitrag zur Begriffsbildung bedarf eines Vokabulars zum Thema, das es erlaubt, sinnhaltig darüber zu kommunizieren. Begriff wird dabei unterschieden von Bezeichner, also dem Begriffsnamen oder -wort, und von Objekt, also einer konkreten Darstellung des Begriffs in (s)einem Anwendungskontext. Die Relationen zwischen Begriff und Bezeichner werden durch Bezeichnung und Bedeutung beschrieben, jene zwischen Begriff und Objekt durch Konkretisierung und Abstrahierung. Zusammenfassend wird dies üblicherweise in einem semiotischen Dreieck dargestellt (Abbildung $1)$.

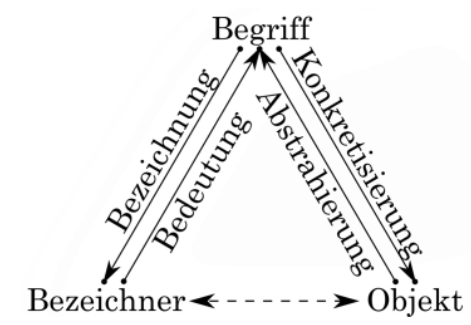

Abbildung 1: Semiotisches Dreieck

\section{Mehrdeutigkeiten im semiotischen Dreieck und das semiotische Dreiecksprisma}

Die Beziehungen zwischen Begriff, Bezeichner und Objekt sind allerdings in vielen Fällen nicht so eineindeutig, wie durch das semiotische Dreieck suggeriert wird. Insgesamt resultieren die Mehrdeutigkeiten daraus, dass jeweils derselbe Begriff, Bezeichner oder dasselbe Objekt, beziehungsweise zwei oder alle drei Komponenten, Bestanteil mehrerer semiotischer Dreiecke sind, die sich überlagern.

Die sich überlagernden und dabei wechselwirkenden semiotischen Dreiecke sollen als Begriffsfeld bezeichnet werden. Das Begriffsfeld kann in Form des semiotischen Dreiecksprismas, an dessen Ecken mehrere, hier der Übersichtlichkeit wegen jeweils zwei Begriffe, Bezeichner und Objekte stehen, modelliert und visualisiert werden (Abbildung 2, nächste Seite).

\section{Begriffsbild und Begriffskonvention im Begriffsfeld}

Das durch einen semiotischen Blickwinkel entstandene Begriffsfeld kann mit seinen verschiedenen Mehrdeutigkeiten Begriffsvorstellungen von Lernenden beschreiben, wobei dort nicht immer alle in dem Dreiecksprisma zu In J. Roth \& J. Ames (Hrsg.), Beiträge zum Mathematikunterricht 2014 (S. 963-966). Münster: WTM-Verlag 


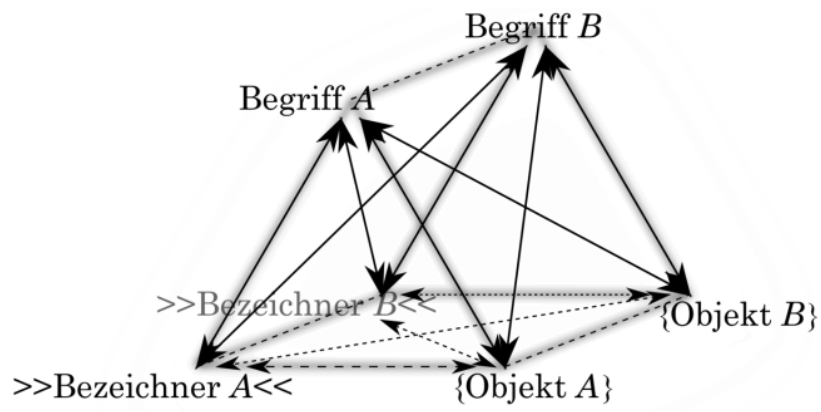

Abbildung 2: Begriffsfeld in Form des semiotischen Dreiecksprismas ${ }^{1}$

findenden semiotischen Dreiecke aktiv sind, und die Beziehungen zwischen den Ecken weniger ausgeprägt sind. Aus der Sicht der Fachdidaktik bleiben diese Begriffsvorstellungen allerdings in Beziehung zu einer fachmathematischen Definition der Begriffe zu setzen. Dazu habe ich auf TALL und VINNERs Concept Image und Concept Definition, sowie, um diese beiden Begriffe zu präzisieren, auf Vorläufer dieser in Philosophie (bei KANT, Frege, CAssirer und Wittgenstein) und Psychologie (bei Piaget, BrUNER, GOODNOw \& AUSTIN sowie ROSCH), und auch auf Arbeiten aus der Fachmathematik stammender Autoren (POINCARÉ, HADAMARD, WITTENBERG und FREUDENTHAL) zurückgegriffen (siehe hierzu: Rembowski 2014a). Schließlich lassen sich die Begriffstypen unter den von mir mit $>>$ Begriffsbild $<<$ und $>>$ Begriffskonvention $<<$ bezeichneten Begriffen zusammenfassen. $>>$ Begriffsbild $<<$ greift dabei zurück auf den Bezeichner von TALL und VINNER, $>>$ Begriffskonvention $<<$ unterstreicht, dass der fachmathematische Begriff intersubjektiv ist und damit konventionalen Charakter hat. Für die beiden Begriffe gilt schließlich:

$>>$ Begriffsbild $<<$ $>>$ Begriffskonvention $<<$

\begin{tabular}{ll}
\hline subjektiv, intuitiv & intersubjektiv \\
\hline synthetisch, induktiv gebildet & analytisch, deduktiv gebildet \\
\hline $\begin{array}{l}\text { gebunden an Bezeichner und kon- } \\
\text { krete Objekte }\end{array}$ & $\begin{array}{l}\text { unabhängig von Bezeichner und } \\
\text { konkreten Objekten }\end{array}$ \\
\hline unscharf & $\begin{array}{l}\text { eindeutig (in Oberklasse und spezifi- } \\
\text { schen Merkmalen) }\end{array}$ \\
\hline
\end{tabular}

kann Handlungen beinhalten, kann blendet den Menschen aus affektiv geprägt sein

unbegrenzt, sich ständig in Ent- klar begrenzt wicklung befindend

${ }^{1}$ Bezeichner sollen nun in spitzen Klammern, Objekte in Mengenklammern stehen. Außerdem werden zwei entgegengesetzte Pfeile zu einem Doppelpfeil verschmolzen. 
Begriffsbild und Begriffskonvention lassen sich nun im Begriffsfeld lokalisieren. Das Begriffsbild enthält ein durch unklare Beziehungen zu charakterisierendes Begriffsfeld, die Begriffskonvention ist eine ausformulierte Zuordnung des Begriffs zu Bezeichner und Objekt (Abbildung 3).

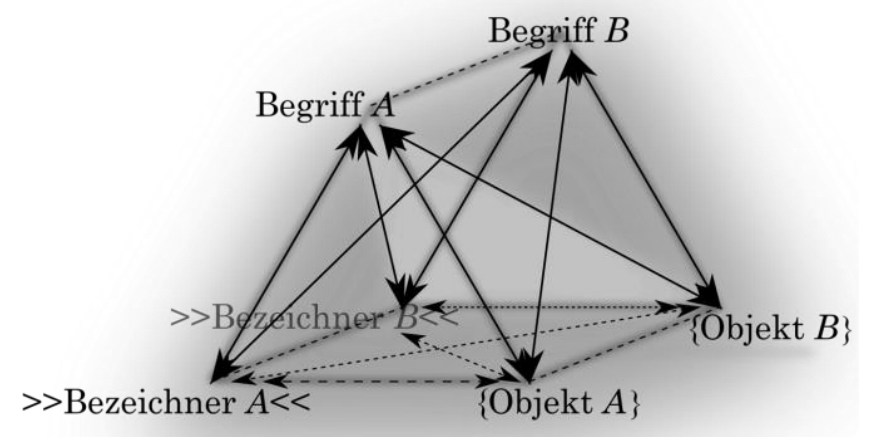

Abbildung 3: Begriffsbild und Begriffskonvention - hell hervorgehoben dargestellt - im Begriffsfeld

\section{Begriffsbild und Begriffskonvention vs. Grundvorstellungen}

Begriffsbildung darf allerdings nicht ausschließlich auf Definitionen beruhen, vielmehr muss zwischen Begriffsbild und Begriffskonvention vermittelt werden. Die vermittelnde Rolle können in der Mathematikdidaktik Grundvorstellungen übernehmen. Um zu klären, was Grundvorstellungen sind/sein sollen, habe ich vor allem auf Arbeiten von BENDER und VOM HOFE zurückgegriffen (siehe hierzu: Rembowski 2014b). Insgesamt sollen Grundvorstellungen intersubjektiven, normativen Charakter haben und auf einer didaktischen Reflexion basieren. Für Lernende sollen sie somit auf im Mathematikunterricht vermittelten Denk- und Handlungsmustern beruhen, und sich zu einem System mentaler mathematischer Modelle zusammenfügen. Grundvorstellungen sollen dabei vielseitige Darstellungen unterschiedlicher Facetten des Begriffsinhalts und gegebenenfalls des Spannungsfelds unterschiedlicher Konkretisierungen sein und diese reflektieren.

\section{Der Würfel}

Zu dem als $>>$ Würfel $<<$ bezeichneten elementargeometrischen Begriff gehört zunächst ein eindeutiges semiotisches Dreieck (Abbildung 4).

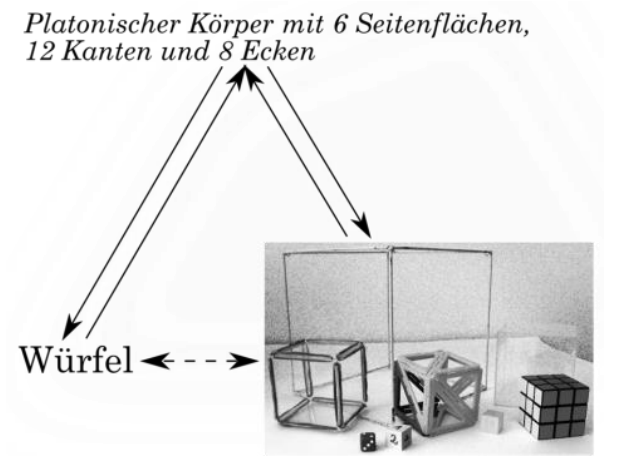

Abbildung 4: Würfel im semiotischen Dreieck 
Die verschiedenen in 1. angesprochenen Mehrdeutigkeiten lassen sich allerdings vollständig auf das Beispiel des Würfels übertragen. ${ }^{2}$ Eine empirische Erhebung in Form der Beantwortung einer offenen Frage in sechsten Klassen an Gesamtschulen hat gezeigt, dass sich viele der Mehrdeutigkeiten tatsächlich im Begriffsverständnis von Lernenden widerspiegeln - es finden sich in wechselnder Zusammenstellung mathematische und alltägliche Begriffe, Bezeichner und Objekte. Insbesondere fällt auf, dass vor allem Begriffe und Objekte mathematisch und alltäglich aufgefasst werden, sowie dass Begriffe und Objekte sehr stark miteinander verwoben sind.

Als Instrument, solche Mehrdeutigkeiten im Begriffsbild und auch Fehlvorstellungen zu nivellieren, können Grundvorstellungen dienen. Grundvorstellungen für den Würfel als Objektbegriff lassen sich dabei formulieren, wenn die verschiedenen Aspekte der Begriffsbildung, die nach VOM HoFE (1995) durch die Grundvorstellungsidee charakterisiert werden, auf den Würfel bezogen werden. So können als Sach- oder Handlungszusammenhang bzw. Handlungsvorstellung des Würfels beispielsweise Aspekte des Gebrauchs von Würfeln im Alltag, Arten der Herstellung von Würfeln und mögliche Operationen mit dem Würfel thematisiert und reflektiert werden. Genannte Punkte werden auch bei der Anwendung des Begriffs auf die Wirklichkeit und dem Modellieren von Sachproblemen wieder relevant, visuelle Repräsentationen bzw. „Verinnerlichungen“ dienen bei geometrischen Objektbegriffen wie dem Würfel allerdings mehr einer prototypischen Begriffsbildung als dem Aufbau von Grundvorstellungen.

Ein Blick in das Schulbuch Mathe Live 5 (Kliemann 2006), mit welchen befragte Lernende gearbeitet haben, zeigt schließlich, dass dort nur die wenigsten, für den Aufbau von Grundvorstellungen zu Würfel relevanten Aspekte, thematisiert sind. Das Schulbuch kann damit für Mehrdeutigkeiten und Unschärfe im Begriffsbild mitverantwortlich gemacht werden.

\section{Literatur}

Eine genaue Auflistung der verwendeten Literatur findet sich in:

Rembowski, V. (2014a) „Concept Image und Concept Definition der Mathematikdidaktik von „Concept Image and Concept Definition in Mathematics“. In U. Kortenkamp \& A. Lambert (Hrsg.): Verfügbare digitale Werkzeuge im Mathematikunterricht richtig nutzen. (im Druck). Hildesheim: Franzbecker.

Rembowski, V. (2014b) „Begriffsbilder und -konventionen in Begriffsfeldern: Was ist ein Würfel?" In A. Filler, A. Lambert \& M. Ludwig (Hrsg.): Geometrie zwischen Grundbegriffen und Grundvorstellungen. (im Druck). Stuttgart: Springer.

\footnotetext{
${ }^{2}$ Dieser Begriff wurde hier gewählt, da er sowohl im Alltag verwendet wird als beispielsweise auch stochastischen Inhalt haben kann, und somit besonders gehaltvoll ist.
} 